\title{
The new hybrid rule-based tool to evaluate processes in manufacturing
}

\author{
A. Macioł · P. Macioł · St. Jẹdrusik • J. Lelito
}

Received: 7 April 2014 / Accepted: 27 January 2015 / Published online: 8 March 2015

(C) The Author(s) 2015. This article is published with open access at Springerlink.com

\begin{abstract}
The aim of our study was to verify if rule-based reasoning systems could be successfully applied for integration of different models and tools which are used for solving complex decision problems in technological design. Engineering problems are unique and differ from typical problems in the domain of management. To solve those problems, an iterative search of the most suitable solutions must be carried out. This puts a pressure on researchers to utilize optimization techniques in connection with simulation methods. Our research is focused on the usage of rule-based reasoning systems for solving simple decision problems, and their integration with highly-specialized tools. This research has been conducted at one of metal foundries, within the scope of supporting technological decisions. A dedicated platform was used in the research, which is tailored for such applications, i.e., Business and Technological Rules Management System REBIT. REBIT system has been developed at the Faculty of Management at the AGH University of Science and Technology in Cracow. In this paper, we prove that the rule-based approach could provide means to integrate all stages of technological
\end{abstract}

\footnotetext{
A. Macioł · St. Jędrusik

Faculty of Management, AGH University of Science

and Technology, ul. Gramatyka 10, 30-067 Kraków, Poland

P. Macioł $(\bowtie)$

Faculty of Metals Engineering and Industrial Computer Science, AGH University of Science and Technology, al. A. Mickiewicza 30, 30-059 Kraków, Poland

e-mail: pmaciol@agh.edu.pl

J. Lelito

Faculty of Foundry Engineering, AGH University of Science and Technology, al. A. Mickiewicza 30, 30-059 Kraków, Poland
}

design. This kind of approach has been successfully applied in the Business Rules Management Systems.

Keywords Rule-based reasoning · Technology designing $\cdot$ Finite element method $\cdot$ Casting process

\section{Introduction}

IT solutions are becoming increasingly popular in the areas of economic activity which were deemed to be the preserve of humans-managers and engineers. This involves solving complex decision problems related to planning activities, customer service, logistics management, product design, or technological issues. All these problems are characterized by multistage complexity, plurality, and diversity of factors that contribute to the solutions. They also impose usage of some hardly measurable parameters which define the way in which decisions are made. As a result, employing IT solutions to solve complex business and technological tasks forces the user to apply various tools, and he then has to solve partial problems relying on his own experience, knowledge, and intuition.

A good example is the finite element method (FEM), which is a powerful tool to evaluate processes in manufacturing. In order to achieve good product quality and process reliability, FEM analyses have to be performed "by hand" repeatedly, with different combinations of process parameters. Meanwhile, it is a very difficult task for engineers to take into account many parameters associated with complex problems, since FEM procedure is very time-consuming and relies heavily on the experience of its users.

An appropriate use of above-mentioned tools is an important issue. Advanced numerical, operational business management, soft computing, and artificial intelligence methods 
constitute the basis for these tools. Defining valid initial and boundary conditions, as well as maintaining the control over model execution, are crucial for achieving reliable results. An iterative search for the acceptable (but rather not optimal) solution is the most popular approach. Hence, the decision-making process is time- and cost-consuming, even when IT tools are involved. Moreover, the validity of final results depends strongly on the quality of decision makers work. In some cases, a concurrent use of several tools is necessary (e.g., in applications using numerical models and soft computing). As a consequence, sharing data between these tools becomes an important issue, since in most cases, the data formats are not compatible with one another. It might happen that a single task can be solved with the use of several IT tools, which differ in time consumption, credibility, and accuracy of solutions they provide. The choice of a suitable solution - for a given case-is within the competence of the user, who is often deprived of any possibility to verify the decision he makes. It may lead to an unnecessary lengthening of the decision-making process or making wrong decisions if an inappropriate tool is applied. Many researchers attempt to solve this problem by integrating different tools, each with diverse features, into a single tool in order to solve complex decision problems. In general, there are two families of such solutions: hybrid tools coupling several sub-models and shell solutions supporting reasoning processes.

The scope of our research was an integration of a rulebased reasoning system with highly-specialized IT tools and models for the purpose of solving complex decision problems in business management. The casting process has been chosen as an example in the field of manufacturing technology. The set of tasks formulated above requires a flexible and easily-extensible rule management system, characterized by an expressive language of knowledge representation. We used REBIT System, which is a Business and Technological Rules Management System that has been developed at the Faculty of Management at the AGH University of Science and Technology in Cracow.

\section{Integration of methods in solving technological problems-literature review}

A fundamental technical problem that arises during planning of the manufacturing process for a product is difficulty in predicting material behavior during final forming. In manufacturing practice, it is generally not possible to conduct a detailed research on physical models due to high costs and time constraints. Therefore, when designing a technology, engineers apply the knowledge in form of guidelines, regulations, and rules that allow for a simulation of material behavior on the basis of numerical models. In the second case, the basic tools that support designers are computeraided design (CAD) and computer-aided engineering (CAE) Many attempts were made to systematize the engineering knowledge in a way so that computers could be used to support the technology design processes. FEM-based numerical modeling methods extend the set of tools that support designers in predicting material behavior during the production stage.

Numerical methods used for modeling are capable of calculating variables such as velocities, stresses, pressures, and temperatures. Calculations are made on the basis of initial and boundary conditions, material properties (liquid/solid metal, mould material), and geometry of the model. They cannot be directly used to calculate a goal function. Nowadays, results are usually analyzed by an expert. It is, however, possible to establish some scalar measures which allow for automatic optimization. Solving well-defined partial differential equations (PDEs) gives comparatively reliable results. However, designing and solving a valid numerical model is time-consuming. While the rate of computations can be improved by increasing the computing power (including parallel computing), the design process must be carried out by an engineer. Furthermore, it is possible that the computations will fail or the results will be deemed to be unreliable. PDEs based solutions can be partially replaced with empirical, simplified, or black-box models, e.g., neural networks $[5,15]$. They are not as reliable as PDEs solutions and not always available, but significantly faster. They compute scalar values instead of using variables and could be easily used to calculate goal functions.

In the literature, there are two substantially different approaches to integrating self-contained support tools for making complex decisions. The first one involves supporting numerical simulations with optimization methods. It is not an integration in the true sense of the word, as it does not cover all phases of the designing process. Nevertheless, this approach covers the key-phases which require the most effort. The FEM and optimization techniques have been integrated in many studies to find the optimal parameters of a metal forming process by transforming a multi-objective problem into a single-objective problem. An example of such a solution is a multi-objective optimization of a sheet metal forming process [18], which combines a simulation based on the FEM with soft computing methods (genetic algorithm in this case). Similar solution had been also designed for multi-objective problems in supplier selection, selecting the most appropriate Flexible Manufacturing System for manufacturing organizations, performance of a manufacturing or service industry system, plant layout design problems, and many others [4]. In [16], an approach based on genetic algorithm (GA), swarm intelligence optimization and FEM were implemented in order to model 
and optimize the process conditions of Al matrix nanocomposites. An example of coupling FEM with multi-objective robust optimization method in sheet metal forming is presented in [17]. In the paper of Macioł et al. [12], the numerical simulation of velocity field of steel flow in the tundish was coupled with the multicriteria optimization procedure. The positions of dams in the tundish were independent variables, while the goal function was described with three properties of the liquid metal flow, computed with the FEM model. The aim of the optimization was searching of the Pareto-optimal set of the geometric parameters of the tundish.

The second approach to the problem of integrating design support methods employs expert systems for this purpose. Expert systems utilize the declarative knowledge. However, several authors present applications of controlled numerical simulations. Replacement of the design procedures carried out by engineers with an intelligent system for press control in a sheet stamping process of trapezoidal panels is described in [8]. Using a database and a FEM-assisted intelligent press control system, the process control path was determined without assistance from an engineering expert, and high efficiency of the process control system was confirmed. The same approach is applied in an expert system for simulating metal sheet stamping. The knowledge base contains decisions that control the FEM. The expert system is used to search for the technological process parameters. All the decisions are accessible via the user interface of a windows-based application that allows to conduct the process analysis automatically [3]. Utilization of an integrated CAD/CAE/CAM system for high pressure die casting is described in [19]. In this paper, a combined expert system is described, which uses a technological database for initial technological design, and the FEM for verification of results. Brown et al. [1] described a web-enabled repository system supporting distributed development of automotive components. The repository employs EPISTLE generic entity modeling principles. Component design and analysis of representational models are referenced as resources through document meta-data, accessible via the virtual repository. A popular approach involves supporting the design of forged parts on the basis of geometry of machined parts, with the help of FEM [7, 14]. Systems that are based on a principle of finding similarities between a designed technology and technologies stored in the knowledge base can also be found [6]. Another category of expert systems is suitable for supporting the design of individual processes. In this case, FEM is used for validation of inference results [2].

In order to effectively support a design processes, it is necessary to determine appropriate data resources. Research works in this field focus mainly on constructing some kind of a technical-technological ontology that is used for exchanging information in automated systems. Similar problems, related to building ontologies for products and technologies, are encountered in the systems that automate the choice of technologies and production plans [9]. Another field where ontologies are employed for supporting the exchange of information is a collaborative technological problem solving. In [13], works are being undertaken by Brunel University in conjunction with the UK's metal forming industry to construct a web-enabled database of material properties to be used in metal forming simulations. An attempt was also made by the authors of this article to generalize the concepts of process modeling and casting products and represent them in the form of an ontology [11]. In the paper of Macioł\& Macioł, the solution suitable for representing complex technical knowledge as a direct combination of a rule-based system and a database inference system that utilizes SQL mechanisms is presented[10]. An exemplary application is described, it is employed to aid in the design of manufacturing process stages and selection of a workpiece. The system was used to answer the following questions: is manufacturing of the particular part possible in given conditions and which forging technology should be chosen. It also allowed for approximation of total production costs.

In none of the above-listed works, authors propose a solution for simultaneous solving of technological and business problems. Indirectly, some business conditions are taken into account in the solutions based on expert systems, such as constraints for eliminating excessively costly or risky products. Nevertheless, dynamic business circumstances (such as availability of raw materials, tight deadlines, etc.) are not considered.

\section{Problem formulation}

One of the cases where business and technological problems should be solved concurrently is fulfilling individual orders. It is a relatively frequent problem across various branches of industry. A technology design process for a newly developed product is used as an example in this paper. This process is carried out at a foundry which is fulfilling an individual order. Process engineers have to design a technology for this order in a relatively short period of time. The procedure for such order is carried out in two stages. In the first step, an evaluation should be conducted to asses feasibility of fulfilling the order. If the result of such assessment is satisfactory, the correlated time requirements and costs should be estimated. At this stage, technologists can (and should) support themselves with expert systems, in order to look for similar technologies that are stored in the knowledge base. The decision making diagram is presented in the Fig. 1. 


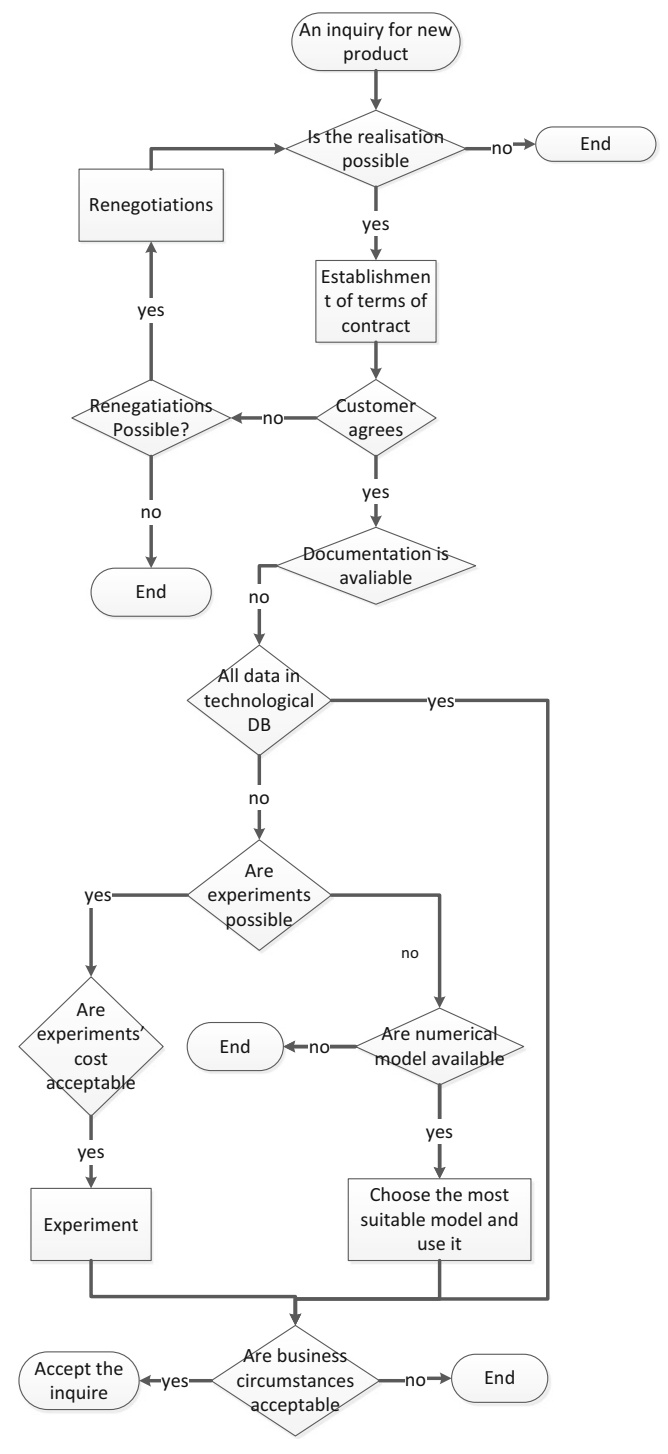

Fig. 1 Decision diagram

Once the feasibility of the order is confirmed and the requirements specified by the customer are acceptable, it is necessary to proceed with the technology development. Initially, the project development is carried out with the use of $\mathrm{CAD} / \mathrm{CAM}$ tools to do 3D solid modeling. As a result, some parameters can be defined such as the gating system, overflow and cooling channels, the gate velocity, feeders, chills, the filling time, etc.

The initially developed casting technology can be used to achieve the production target. However, there might be some circumstances which will require taking additional actions. Firstly, the technological database may be lacking suitable data and/or examples, so a reliable solution to the design problem cannot be found. Secondly, economical or market circumstances may lead to verification of the preliminary initial. Such situations are acceptable in cases when costs of achieving the production target are high and/or very high qualitative requirements are specified by the client. In all those cases, engineers have different solutions at their disposal. The best results can be achieved by carrying out production trials for different variants of technological solutions. Unfortunately, this is the most expensive and time-consuming approach that can be only very rarely. Similar results can be achieved by utilizing hybrid systems for optimization of the technological processes, as described in the Section 4. However, these systems are highly specialized and as such are not widely available. Their use in the business management of enterprises is very limited.

If it is necessary to verify the initial decisions, engineers tend to most frequently use relatively easily available tools (CAP of EKK, FLOW-3D of Flow Science, Pro/CAST of UES, USA, and MAGMASOFT of Magma, Germany, etc.) [19] to simulate only some chosen potential problems. These tools can be used to model processes related to the flow of liquid metal, solidification and crystallization, cooling, heat transfer, segregation, convection, heat treatment, and many others (Fig. 2). However, obtaining a reliable results is difficult. High skills of users, as well as reliable and detailed knowledge base of material properties are crucial. Due to the time-consuming nature of such studies and their relatively high costs (especially in case of outsourcing or software leasing), simplified solution can be also applied. They are most frequently based on the soft computing methods. Of course, the simulation studies are focused on the most important phenomena that take place during the process of casting. The direction of research depends on (described in the literature or internal reports of producers) the most likely faults that cause defects. The procedure of simulation and evaluation of its results is usually realized iteratively until one obtains satisfactory result. The result of simulation study is the final form of casting production project.

Numerical modeling and results verification are usually executed in an iterative loop until satisfactory results are achieved. Those results are then used as a final form of casting procedures.

The decision-making process is tightly coupled with technological models and experimental results. For every business decision, some technological questions must be answered (can a given order be manufactured, how complicated it is, etc.). On the other hand, while making technological decision some business circumstances have to be taken into account (are raw materials available, are any surrogate materials available, how important order is, what are the deadlines, etc.). In a traditional decision-making process (Fig. 1), it is quite difficult to strike a balance between the response time to a customer's inquiry, the response preparation costs (mainly costs of the experiments), and the risk inherent to the manufacturing process. 


\subsection{Proposed solution}

In the preceding sections, two important issues were emphasized: (i) the traditional way of order fulfilment is inefficient, and (ii) in the production preparation process business circumstances should be taken into consideration. Neither expert systems used for supporting business decisions nor numerical simulations tools are capable of addressing both these issues concurrently. In this paper, we would like to describe a solution based on REBIT System, which is presented below.

A general scheme of cast metal production planning integrated system is shown in the Fig. 3.

Research works in the area of supporting technological decision have been conducted at one of the branches of a metal foundry. The essence of the proposed solution lies in a parallel execution of tasks which are managed by the internal rule-based system. These tasks include:

a

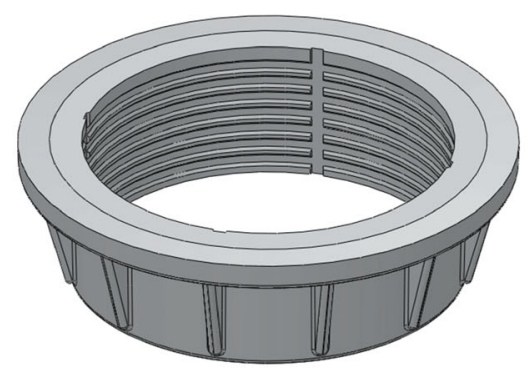

C
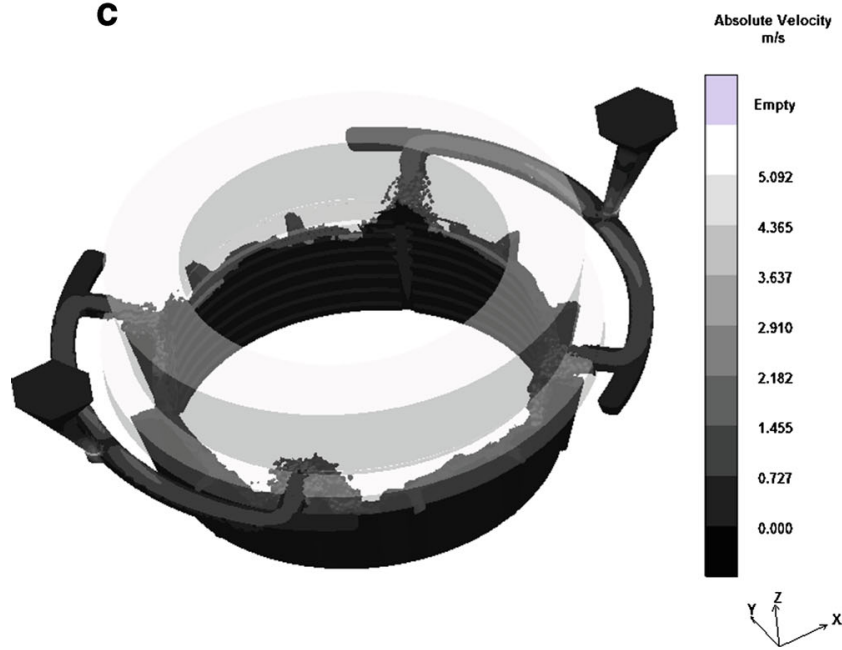

- Reasoning in a declarative knowledge base

- Selecting suitable tools for solving problems which require usage of sophisticated models (e.g., FEM models)

- Selecting input parameters for the IT tools

- Supervising the iterative search of satisfactory partial solutions

Additionally, it was assumed that the integrated rulebased system would be employed to solve operational business management problems such as production failures (e.g., defects, failed, or misfit charges) or issues caused by external influences (e.g., user ordering a novel product). In both cases, a quick solution is expected, while the availability of resources and tools may be limited. As a result, rule-base hybrid system must be able to take into account some restrictions and criteria resulting from the current operating conditions of the enterprise.

b
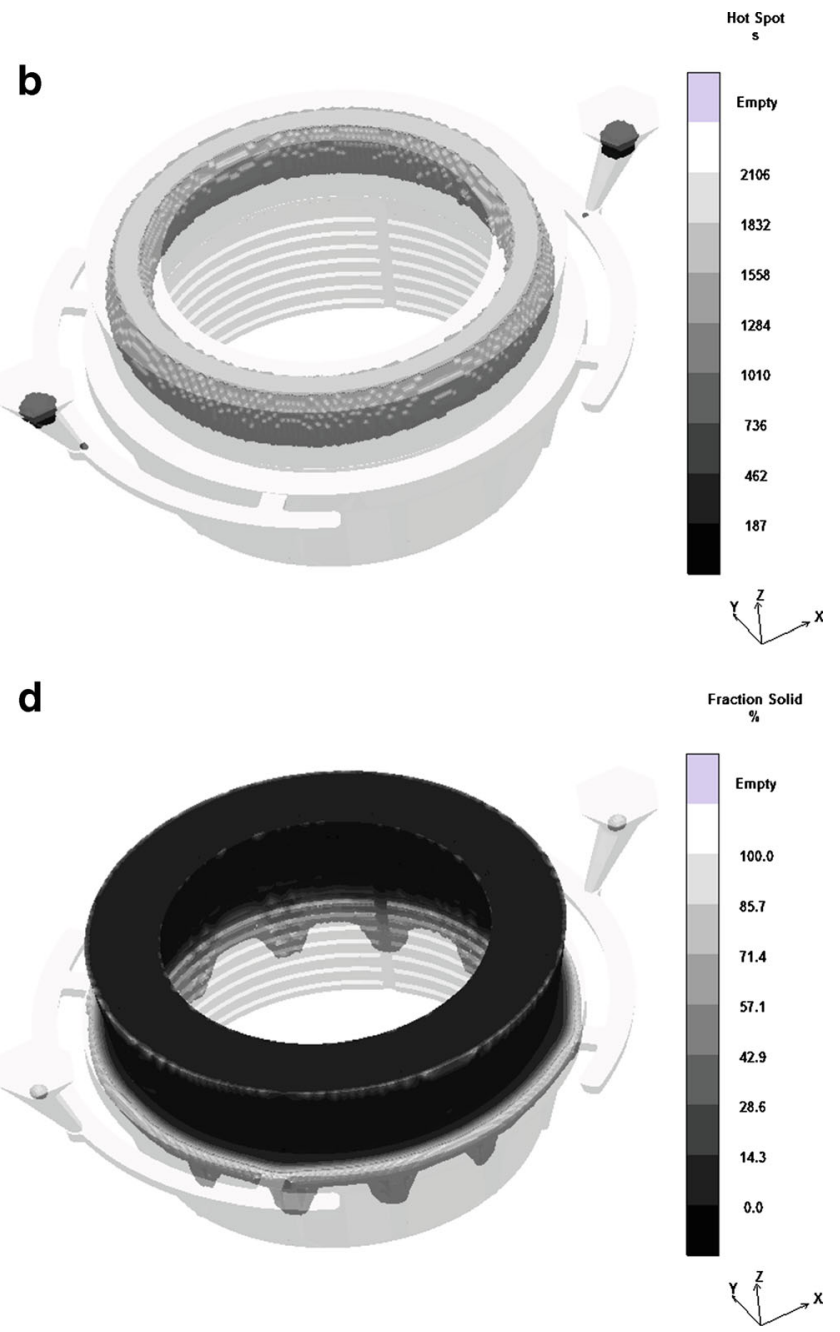

Fig. 2 An exemplary application of simulation tools (MAGAMSOFT) for the casting process design. 3D CAD model (a); hot spots (b); filling of the mould cavity (c); solidification (d) 

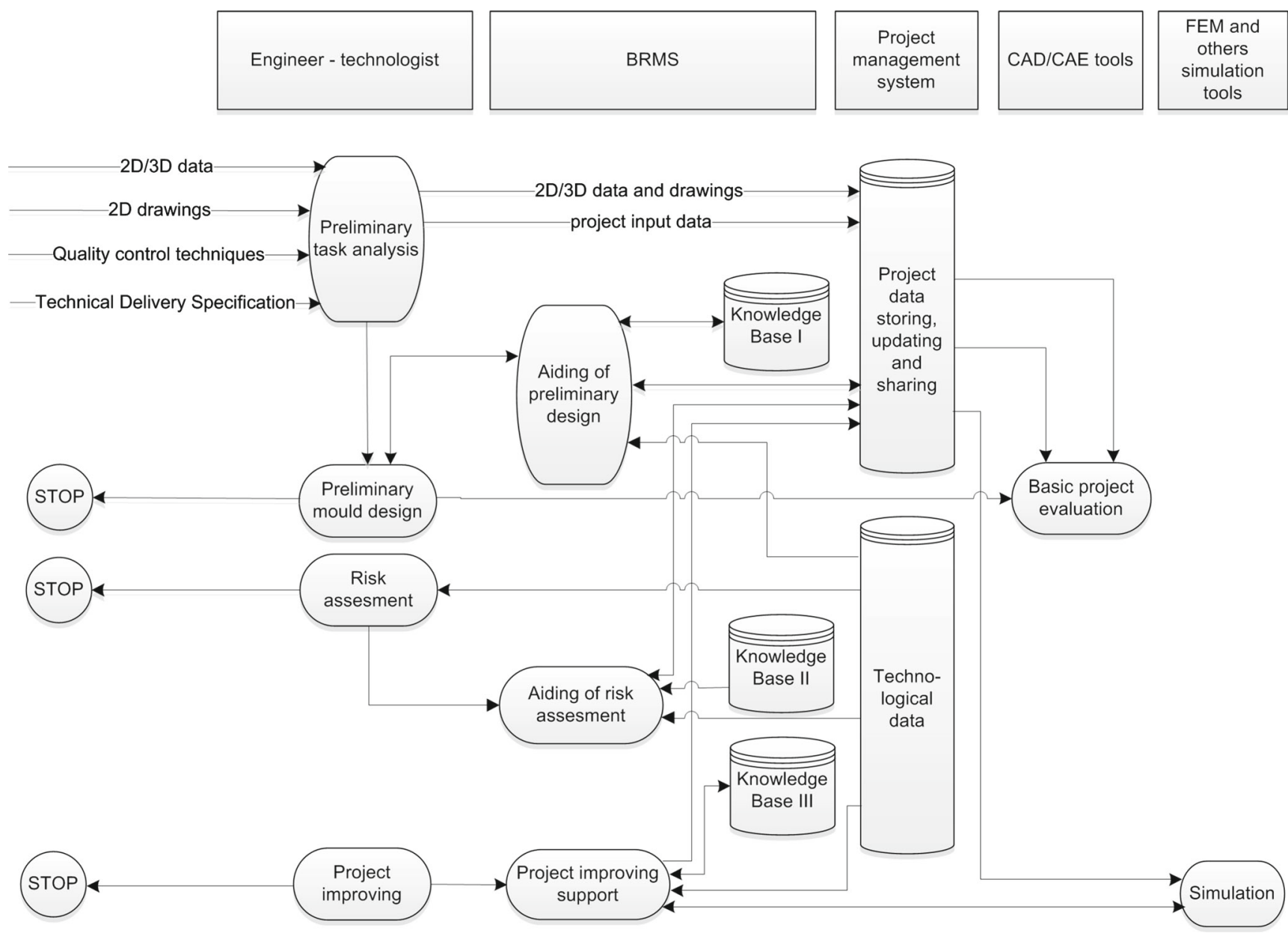

Fig. 3 The general scheme of proposed solution

The proposed solution will be shown on an example of an enquiry handling procedure: a client wants to manufacture a small series of metal casings. Descriptions of the most important procedures as well as knowledge required to configure and run specialized tools are presented in this paper. Reasoning mechanism specifications and software requirements are also discussed.

\section{Business and technological rules management system rebit}

As it was mentioned above, a perquisite for a successful utilization of the hybrid system in the multi-scale modeling of technological processes is an effective combination of a rule management system and a process modeling system (e.g., FEM). The essence of this approach lies in creation of a platform for undistorted exchange of information among the rule-based system and simulation models. It is also necessary to use such rule-based systems that are capable of identifying modeling requirements and interpreting simulation results. This is due to the fact that these systems are general purpose systems with an universal syntax. They are not equipped with specialized interfaces to cooperate with technological simulation models. The only BRM system that was designed for technological rules is REBIT system.

Rete algorithm can be used to decrease the running time of the inference process up to several orders of magnitude when compared to a naĀŕve implementations of the inference algorithm. It turns out, however, that the running time of an inference algorithm is not the most important criterion in some applications. An example of such a case is the multi-scale modeling of technological processes. Our studies indicate that there are many problem domains where factors other than the running time of an inference algorithm are more important, e.g., the ability of controlling the inference process in such a way to avoid querying for certain variables. This desirable feature of the inference algorithm stems from the fact that in certain problem domains the 
cost of calculating values of some variables is high. The most commonly used rule-based systems that implement Rete algorithm do not take into consideration the cost of obtaining values of the variables. Rete algorithm lacks this feature as well. This was the main reason for development of a new inference algorithm and an entirely new rule-bases system called REBIT. According to our best knowledge, REBIT algorithm is the only one that allows the inference process to take into consideration the cost of calculating values of variables. The inference process is aimed at achieving the final conclusions in the smallest possible number of steps. In the case of knowledge base used in modeling of technological processes, values of certain variables are not directly available. In order to obtain those values, an experiment has to be carried out. Therefore, using different inference paths can be more advantageous, despite the fact that it requires a greater number of queries. REBIT algorithm selects an inference path which is characterized by the minimal cost. The cost of variables has to be determined, which is the additional task performed by a knowledge engineer in REBIT system. Determining the cost of variables can be also regarded as a way to control the inference process. REBIT system supports all stages of knowledge-base development process. Knowledge-base editor is usually the first tool that is used in the process. The editor allows creating and editing entries in a graphical or text-based manner with the help of intelligent prompts. All classic elements of knowledge representation are also available in the REBIT language. Rules, variables, and functions are the main building blocks. The rules are organized in so called rule sets, i.e., groups of logically connected rules. Rule sets may form a stand-alone knowledge base or constitute a part of more a complex knowledge base. The REBIT language provides also more sophisticated elements such as grids and decision tables. Manual testing of a newly created knowledge base can be carried out with the use of REBIT generic client. A technologist can start the reasoning procedure by selecting a knowledge base from a repository. During the reasoning process, the rule engine is capable of querying a technologist for any additional data that is required. The reasoning process can be paused and resumed at any time. When resuming, the engine is able to return to a rule which was being processed before the procedure was suspended. The unique feature of REBIT System is a two-level knowledge model. The bottom level consists of well-known production rules and more sophisticated elements like grids and decision tables. The top level is more algorithmic-like, if consists of workflow-based elements. It is used to represent a procedural knowledge. It includes such elements as gateways, loops, atomic, and structural activities. The two-level model of knowledge is very useful for solving technological problems encountered, e.g., in material science, metallurgy, and other areas. All components of REBIT system may be integrated into a standalone application. However, their main advantage is that they form a set of freely connected components working in service-oriented architecture (SOA). Some of these components act as service providers, while others are the service consumers. The cooperation between REBIT may be perceived as a king of choreography. There is not any central coordinator, so every component knows all necessary details of the collaboration process. All the components may be executed on a single node or - in a fully distributed manner-every one of them on a different node. Every component is an autonomous application with a well-defined public interface. The communication utilizes SOAP protocol over HTTP or TCP. Hence, the REBIT components may interact with any external component which conforms to the SOAP specification. Usage of REBIT system for supporting multi-scale modeling of technological processes is possible owing to the following features:

- The possibility of considering the cost of a query in the inference algorithm

- The possibility of running long-running interface sessions

- The possibility of pausing and resuming the inference sessions, while preserving functionality to restore a previously processed rule

\section{An example of REBIT application}

The concept of supporting the design process with REBIT system is shown on an example below. Problems are considered which are related to the development of a manufacturing technology for the grey iron casting in sand moulds.

In the first phase of the production preparation for a new casting, there are some basic engineering works that require the support. These works involve designing the mould and defining the process parameters. The knowledge that is needed to carry out these tasks is stored in a tabular form, as well as in the form of nomographs, computational formulas, and sets of exemplary solutions for different input conditions and restrictions (e.g., chemical composition of the alloy, overall dimensions, weight, and shape of the casting).

Unique features of the REBIT language and a suitable rule management system allow to store, in a simple manner, the knowledge which is required for designing the mould and the casting process. The knowledge can be stored in the form of SQL tables, decision tables, decision grids, and rule sets. The user can easily apply this knowledge and modify it without necessity to alter the application code. 
In the first phase, a rule set is used as a guide to resources stored in the databases. Some fixed scalar values and relatively simple computational formulas are stored in the rule set as well. An exemplary rule set used for the computation of the solidification time is presented below:

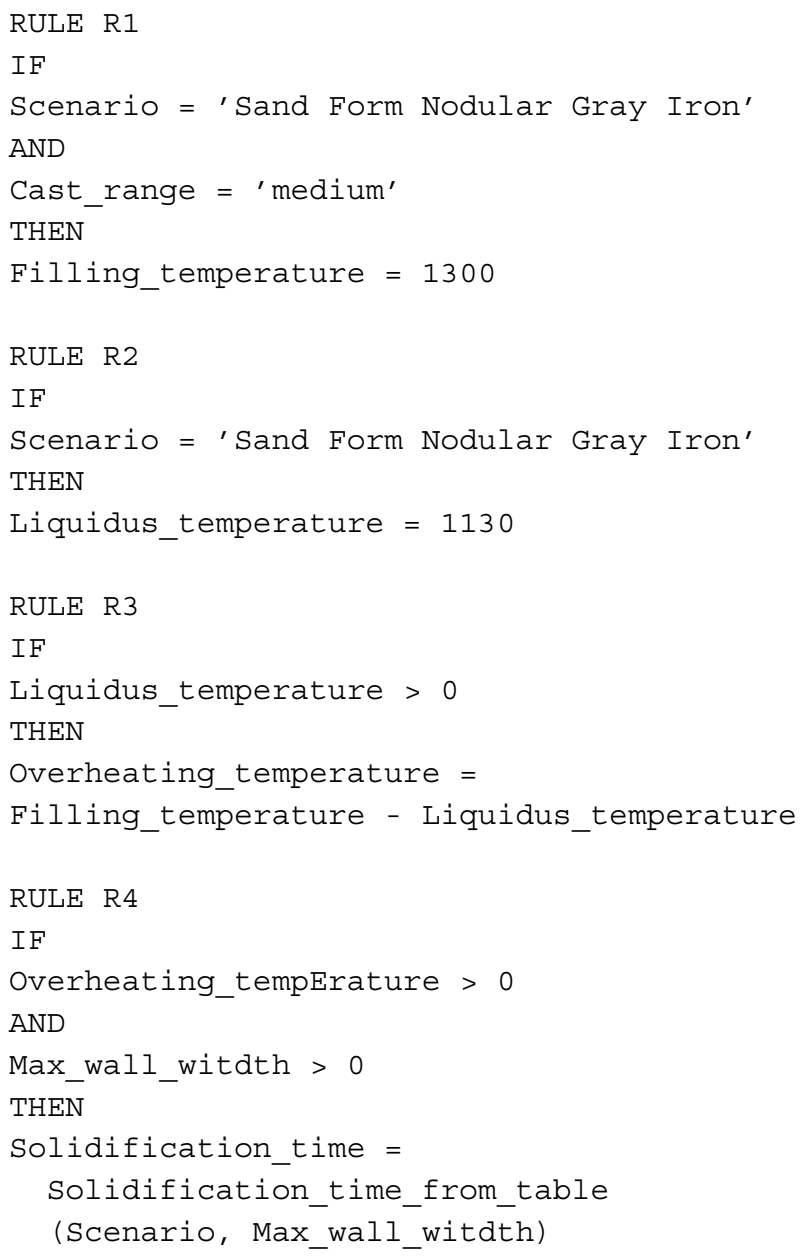

The enumerated variable used in this scenario contains encoded information on the casting technology and the material. The Max_wall_width variable can be introduced by the user during reasoning session, or imported from an external database. The Solidification_time variable is linked to a SQL table via the resource management module. An object of the Query class has a QueryString property and is responsible for retrieving data from the database. The QueryString could be defined for example as follows:

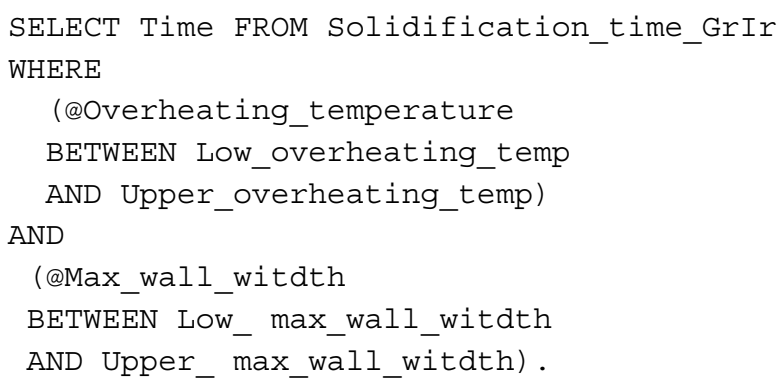

The resources management module is used to replace the terms starting with "@” prefix with the values of the corresponding variables. The query result is linked to a corresponding variable (in this case Solidification_time_from_table) via an object of the QueryBinding class.

When the first stage of design works is completed, a pilot project of the casting production technology is obtained. Concurrently, the designer prepares appropriate drawings with the help of CAD tools.

In the second phase of the design process, a more complex decision-making procedure has to be conducted. A decision-support module that is built on the basis of REBIT system has to answer the following questions:

- Which parts of the "rough" design must be verified?

- Are there any reasons to carry out an industrial-scale research?

- Are there any precise process optimization tools and whether is it economically justified to use them?

- What tools should be used for the fragmentary research?

- Which input parameters should be covered by the fragmentary research and what research method is suitable?

Two modules are used to support decisions in this phase of the design process:

- Preliminary scheme evaluation

- Partial problem solving

The first of these modules is responsible for assessing the necessity of a detailed research. It also has to define the scope of the research and select appropriate research methods. The module relies on the knowledge base that is stored in the form of rule sets, it also utilizes technological databases and research methods.

In the next stage of the process, a more thorough analysis of the adopted solution is carried out, and a potentially suitable research method is selected. For this purpose, a module consisting of a single rule set is used (in case of complex inference processes a few rule sets may be used). To accomplish the task, it is necessary to gather some essential information on the project, as well as on the conditions defined by the order. The assessment of the order and the additional conditions may be accomplished by the designer with or without the help of rule sets. The risk of fault occurrence can be estimated subjectively or the inference engine can be used for this purpose. In the latter case, the inference engine will apply rules to bind variables to parameters such as material type, mould technology, shapes complexity (evaluated by analysing the CAD drawings), etc. Regardless of which procedure is used for determination of those 
values, in the next stage, rules will be applied that are similar to the one presented below.

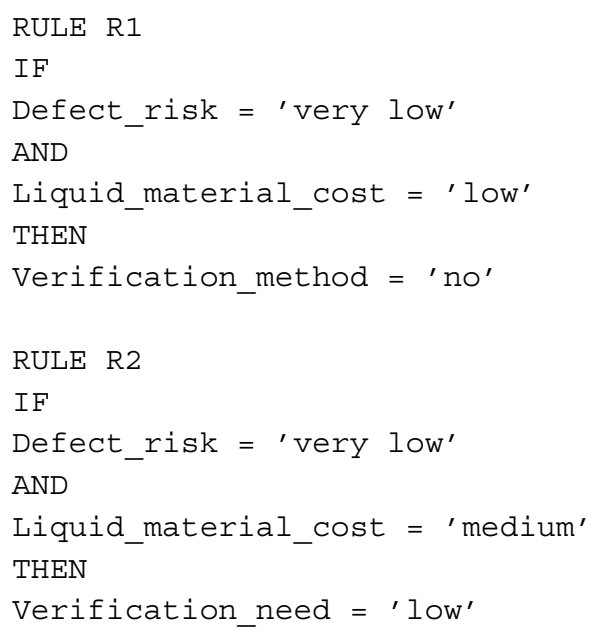

Upon the completion of this stage, results that are acquired will clearly define a direction of further research. They should also define the purpose and the scope of this research and indicate the most effective research methods.

In the next stage, a research is carried out, which is aimed at verifying the "rough" project of the technology. In the following assumed scenario, a technology evaluation indicated that there is a threat of misruns and wash defects, and that a suitable method to address and solve those problems is the FEM simulation. An engineer updated the initial technology description and uploaded a suitably modified CAD drawing to the simulation program, and then started the first run. The simulation results were initially interpreted by the engineer and uploaded to the module which combines experiment control procedures and rule sets. This module was used to conduct an evaluation of the results and to define a direction of changes in the technology.

In this phase, a set of independent components is used. These components are typical simulation tools or tools based on the soft computing principle. The process of obtaining a satisfactory solution is peculiar; therefore, it requires simultaneous usage of declarative and procedural knowledge. REBIT system is capable of dealing with such situations, and it allows to record procedures (including iterations) in the form of workflows. At the same time, rule sets can be embedded as an internal part of this workflow. Such solution has an important advantage when compared to a classic decision support system (DSS). Any modifications (resulting, e.g., from new experiences) can be implemented at the rule-editor level, without the necessity of modifying the code.

A numerical simulation is preceded by the analysis of a CAD drawing. An engineer has to define continuous zones and their mutual locations. Metal flows from a previous zone into an inSite zone. A further procedure utilizes an iterative loop. Reasoning engine is executed every time a numerical solution is being computed, and the rules used are described below. Reasoning sessions are provided for each pair of previous and inSite zones for which failures were anticipated. During each session, the workflow module updates the simulation results and the data from the previous session via a resource management module (e.g., site heat transfer, filling velocity (basing on numerical model), or previous filling velocity (basing on the previous sessions)). Corrected values of mould properties (heat transfer in sites), casting process properties (filling velocity), or mould rearrangement guidelines are the exemplary outputs of a reasoning process.

The rule set which is described below represents an exemplary knowledge base used to search for corrections in the casting technology, in case when misruns are present in the examined zone. Logical relations between the rules are shown in the Fig. 4. Rules R1 to R10 are related to modifications of heat transfer in inSite and previous zones. Decreasing the heat transfer in the inSite zone can lead to elimination of misruns. However, it might happen that the heat transfer cannot be decreased in the inSite zone. Then, a possibility of decreasing the heat transfer in previous zone is examined. If it is not possible, other process properties are 


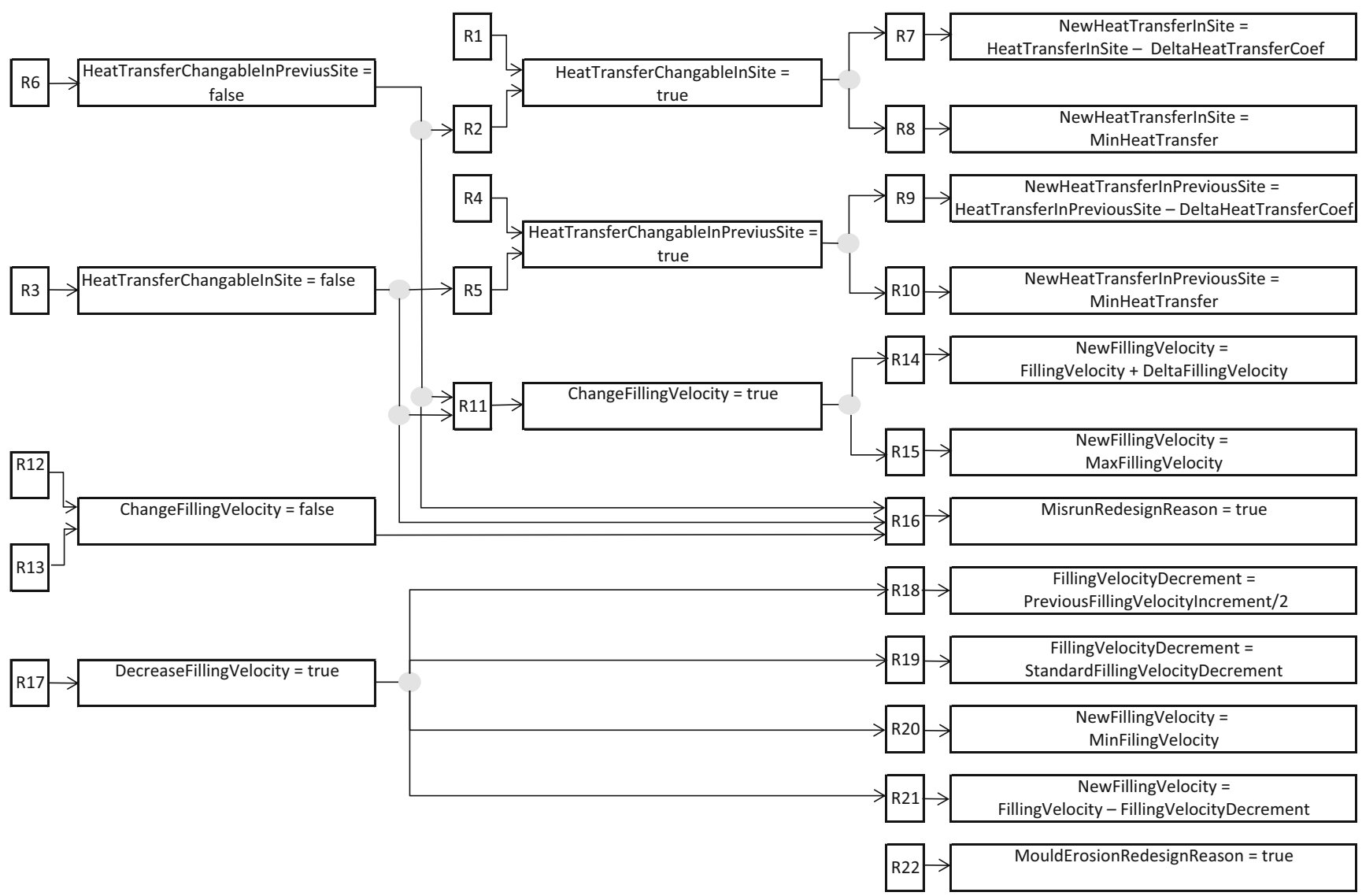

Fig. 4 Logical structure of the knowledge

checked. Rules R11 to R22 are responsible for increasing the filling velocity. When the velocity cannot be increased (e.g., due to the risk of a mould erosion in site, as predicted by the numerical model), a mould redesign is suggested to the engineer.

RULE R1

If Misrun = true

AND

HeatLostInPreviousSite $<=$ HeatLostInsite

AND

HeatTransferInSite > MinHeatTransfer

THEN

HeatTransferChangableInsite $=$ true

RULE R2

If Misrun = true

AND

HeatLostInPreviousSite > HeatLostInSite

AND

HeatTransferChangableInPreviousSite = false

AND

HeatTransferInSite > MinHeatTransfer

THEN

HeatTransferChangableInsite = true
RULE R3

IF

HeatTransferInSite $=$ MinHeatTransfer

THEN

HeatTransferChangableInsite = false

RULE R4

If Misrun = true

AND

HeatLostInPreviousSite > HeatLostInSite

AND

HeatTransferInPreviousSite

$>$ MinHeatTransfer

THEN

HeatTransferChangableInPreviussite

$=$ true

RULE R5

If Misrun = true

AND

HeatLostInPreviousSite $<=$ HeatLostInSite

AND

HeatTransferChangableInSite = false

AND 
HeatTransferInPreviusite

$>$ MinHeatTransfer

THEN

HeatTransferChangableInPreviussite

$=$ true

RULE R6

IF HeatTransferInPreviusSite

$<=$ MinHeatTransfer

THEN HeatTransferChangableInPreviussite

= false

RULE R7

IF HeatTransferChangableInsite = true

AND

HeatTransferInSite - deltaHeatTranferCoef

$>$ MinHeatTransfer

\section{THEN}

NewHeatTransferInSite

= HeatTransferInsite - DeltaHeatTransferCoef

\section{RULE R8}

IF

HeatTransferChangableInSite = true

AND

HeatTransferInSite - DeltaHeatTransferCoef

$<=$ MinHeatTransfer

THEN

NewHeatTransferInSite $=$ MinHeatTransfer

RULE R9

IF

HeatTransferChangableInPreviousSite = true AND

HeatTransferInPreviousSite

- DeltaHeatTransferCoef > MinHeatTransfer THEN

NewHeatTransferInPrevioussite

= HeatTransferInPreviousSite

- deltaHeatTransferCoef

RULE R10

IF

HeatTransferChangableInPreviousSite = true

AND

HeatTransferInPreviousSite

- DeltaHeatTransferCoef <= MinHeatTransfer THEN

NewHeatTransferInPreviousSite

= MinHeatTransfer

RULE R11

IF

Misrun = true

AND

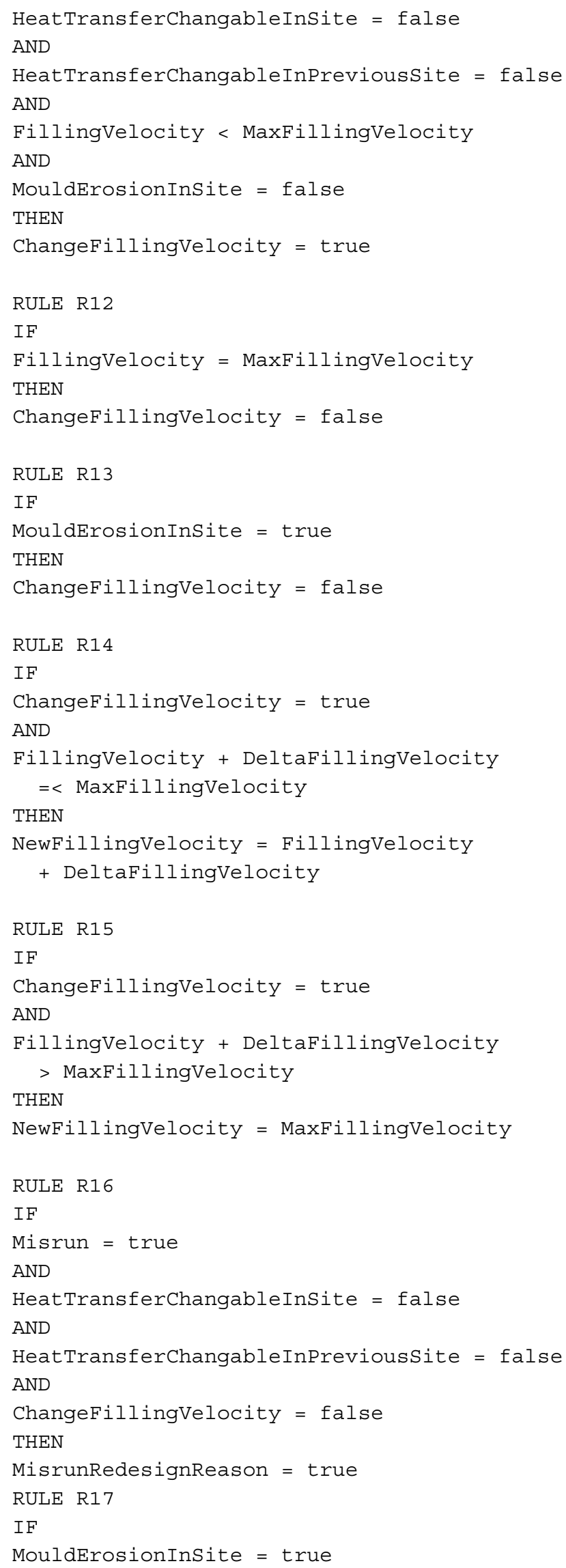




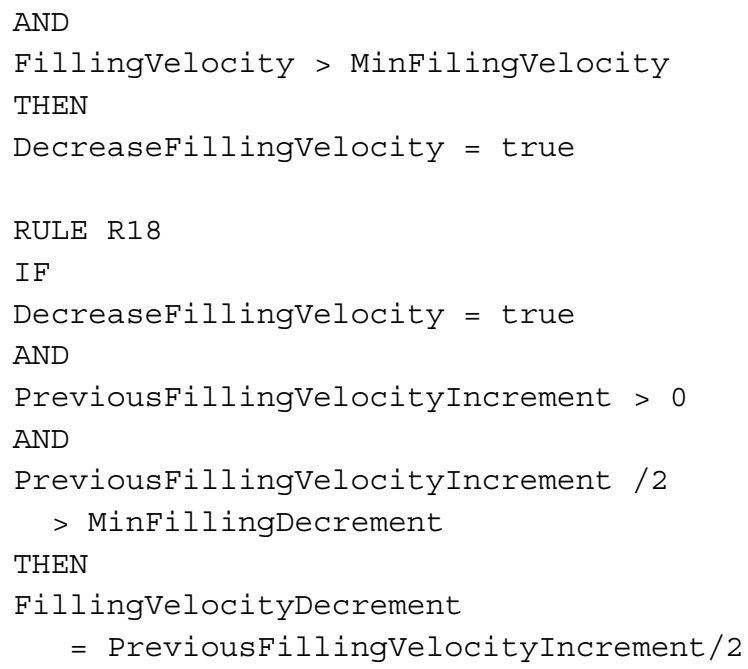

This solution combines procedural, simple-structured knowledge (two nested loops) with easily expandable declarative knowledge. As a result, it allows to develop software which is useful and easy to maintain.

\section{Conclusion and further works}

A solution is proposed, which addresses the problem of integrating IT tools that are used for supporting the design processes at a metal foundry. The research confirmed that whenever a process is complex enough, it cannot be supported with a single IT solution. A full automation of the process is also impossible. Therefore, it should be assumed that an engineer's involvement in unavoidable during every design phase. However, it is possible to develop such a comprehensive software solution which is capable of assisting technologists during every design phase. Such software is not available on the market. In the literature, no propositions on how to develop such a solution can be found. In our opinion, the rule-based approach allows for a comprehensive integration of the design process; this was confirmed by the preliminary research. The approach has been successfully applied in Business Rules Management Systems which are an extension of Business Process Management Systems. Engineering problems are unique and differ from typical decisions that are made in the areas of management. To solve those problems, an iterative search of the most suitable solutions must be conducted. This puts a pressure on researchers to utilize optimization techniques in connection with simulation methods. However, this type of solutions covers only a limited range of tasks, which are usually very different from one another. Therefore, only one suitable solution is known to the authors, i.e., the integration of design phases and support tools during the design process. This is achievable by using workflows to control iterations and rule-based systems. REBIT system, which is described in this paper, has all the necessary features to meet those requirements. Defining the rules for collection and sharing of complex engineering knowledge is a key problem in the effective implementation of the presented concept. The further research will be aimed at creating a suitable ontology, which will be a continuation of the previous works of the authors [11].

Open Access This article is distributed under the terms of the Creative Commons Attribution License which permits any use, distribution, and reproduction in any medium, provided the original author(s) and the source are credited.

Acknowledgements This work is supported by the NCN, project No. 2011/01/D/ST8/04984 


\section{References}

1. Brown D, Leal D, McMahon C, Crossland R, Devlukia J (2004) A Web-enabled virtual repository for supporting distributed automotive component development. Adv Eng Infor 18(3):173-190. http://www.sciencedirect.com/science/article/pii/ S1474034605000042

2. Cakir MC, Irfan O, Cavdar K (2005) An expert system approach for die and mold making operations. Robot Comput-Integr Manuf 21(2):175-183. doi:10.1016/j.rcim.2004.07.015. http:// linkinghub.elsevier.com/retrieve/pii/S0736584504000791

3. Álvarez Caldas C, Quesada A, Gauchía A, San Román JL (2009) Expert system for simulation of metal sheet stamping. Eng Comput 25(4):405-410. doi:10.1007/s00366-009-0137-2

4. Ha SH, Krishnan R (2008) A hybrid approach to supplier selection for the maintenance of a competitive supply chain. Expert Syst Appl 34(2):1303-1311. doi:10.1016/j.eswa.2006.12.008. http:// www.sciencedirect.com/science/article/pii/S0957417406004180

5. Karunakar DB, Datta GL (2007) Prevention of defects in castings using back propagation neural networks. The Int J Adv Manuf Technol 39(11-12):1111-1124. doi:10.1007/s00170-007-1289-0

6. Katayama T, Akamatsu M, Tanaka Y (2004) Construction of PC-based expert system for cold forging process design. J Mater Process Technol 155-156(0):1583-1589. doi:10.1016/j.jmatprotec.2004.04.256. http://www.sciencedirect. com/science/article/pii/S092401360400679X

7. Kim C, Park CW (2005) Development of an expert system for cold forging of axisymmetric product. The Int $\mathbf{J}$ Adv Manuf Technol 29(5):459-474. doi:10.1007/s00170-005-2532-1

8. Koyama H, Wagoner RH, Manabe Ki (2004) Blank holding force control in panel stamping process using a database and FEM-assisted intelligent press control system. J Mater Process Technol 152(2):190-196. http://www.sciencedirect.com/science/ article/pii/S092401360400456X

9. Kumar S, Singh R (2005) An intelligent system for selection of die-set of metal stamping press tool. J Mater Process Technol 164:1395-1401. http://www.sciencedirect.com/science/article/pii/ S0924013605001378
10. Macioł A, Macioł $P$ (2008) Intelligent hybrid system for forging process design support. Steel Res Int 79:521-528

11. Macioł A, Wrona R, Stawowy A, Macioł P (2007) An attempt at formulation of ontology for technological knowledge comprised in technical standards. Archives Metallurgy Mater 52(3):381-388

12. Macioł P, Gawảd J, Podorska D (2007) Arrangement of flow modification devices in continuous casting tundish based on multicriterion optimization. Archives Metallurgy Mater 52(1):105112

13. Mynors D, Broomhead P, Grieve R (2004) Web-based material properties for use with metalforming simulation packages. J Mater Process Technol 153:688-693. http://www.sciencedirect. com/science/article/pii/S0924013604004832

14. Ravi R, Prasad Y, Sarma V (2003) Development of expert systems for the design of a Hot-Forging Process based on material workability. J Mater Eng Perform 12(6):646-652. doi:10.1361/105994903322692439

15. Shabani MO, Mazahery A (2012) Artificial intelligence in numerical modeling of nano sized ceramic particulates reinforced metal matrix composites. Appl Math Model 36(11):54555465. doi:10.1016/j.apm.2011.12.059. http://www.sciencedirect. com/science/article/pii/S0307904X11008675

16. Shabani MO, Mazahery A (2013) Application of GA to optimize the process conditions of Al Matrix nano-composites. Compos Part B: Eng 45(1):185-191. http://www.sciencedirect.com/ science/article/pii/S1359836812004994

17. Sun G, Li G, Gong Z, Cui X, Yang X, Li Q (2010) Multiobjective robust optimization method for drawbead design in sheet metal forming. Mater \& Des 31(4):1917-1929. http://www. sciencedirect.com/science/article/pii/S0261306909006049

18. Wei L, Yuying Y (2008) Multi-objective optimization of sheet metal forming process using Pareto-based genetic algorithm. J Mater Process Technol 208(1):499-506. http://www. sciencedirect.com/science/article/pii/S0924013608000721

19. Yue S, Wang G, Yin F, Wang Y, Yang J (2003) Application of an integrated CAD/CAE/CAM system for die casting dies. J Mater Process Technol 139(1):465-468. http://www.sciencedirect.com/ science/article/pii/S0924013603005065 